Bull. Mater. Sci., Vol. 7, No. 5, December 1985, pp. 499-507. (C) Printed in India.

\title{
Influence of incident beam divergence on the intensity of diffuse streaks
}

\author{
DHANANJAI PANDEY $\dagger$, S LELE ${ }^{*}{ }^{+}$, LALITA PRASAD $^{+}$and \\ J P GAUTHIER** \\ † School of Materials Science and Technology and ${ }^{+}$Department of Metallurgical \\ Engineering, \\ Banaras Hindu University, Varanasi 221005 , India \\ ** Laboratoire de Mineralogie-Cristallographie, Universite' Claude Bernard Lyon I, 69622- \\ Villeurbanne Cedex, France \\ MS received 1 July 1985
}

\begin{abstract}
The influence of incident beam divergence on the length of the streak intercepted by the Ewald sphere is considered, as a relp $H K \cdot L$ of a faulted hexagonal crystal, mounted about its $c$-axis on the goniometer head attached to the $\phi$-circle, is brought into diffracting condition for the bisecting setting of a 4-circle diffractometer. For the special crystal mounting correction factors required to convert the measured intensities corresponding to a fixed length of the streak are derived. A procedure for experimentally verifying the mathematical approach employed in these derivations is also presented.
\end{abstract}

Keywords. Incident beam divergence; diffuse streaks; Ewald sphere; crystal orientation.

\section{Introduction}

A statistical distribution of planar defects such as stacking faults in close-packed structures is known to give rise to characteristic diffuse streaks along certain reciprocal lattice rows parallel to the stacking axis. The theory of diffraction from randomly as well as non-randomly faulted close-packed structures is reasonably well understood and from a comparison of the theoretically predicted diffraction effects with those experimentally observed one can study not only the nature, distribution and concentration of stacking faults (Pandey and Krishna 1977) but also the mechanism of phase transformation occurring as a result of insertion of stacking faults (Pandey et al 1980; Lele and Pandey 1985). Such a study requires an analysis of the continuous intensity distribution along the streaked-reciprocal lattice rows. Using single crystal intensities, it is possible, in principle, to match the experimentally observed intensity distribution along the streak with that calculated theoretically for a model postulating a certain random or non-random distribution of faults in the structure. An analysis of this type can provide information about the defect structure much in the same way as the analysis of Bragg intensities in the determination of regular structures. However, there are special problems stemming from several factors, like incident beam divergence, polychromaticity of the $x$-ray radiation, finite detector slit and instrumental resolution function, in the measurement of the intensity of diffuse streaks using a single

\footnotetext{
* To whom all correspondence should be addressed
} 
crystal diffractometer. A proper understanding of the influence of these factors on the measured intensities and the application of the corresponding correction factors requires specification of the diffraction geometry. In the present investigation, we consider the influence of the incident beam divergence for a hexagonal crystal oriented about its $c^{*}$ axis on the goniometer head. The divergence correction factor for $c$-axis mounting is worked out for the bisecting geometry. An experimental verification of the mathematical approach employed in deriving the divergence correction factor is also presented.

\section{Reference system and crystal orientation}

Let us consider a Cartesian coordinate system $X Y Z$ attached to the diffractometer such that the + ve $X$-axis is directed opposite the incident beam from the centre of the goniometer, the $Z$-axis is directed upwards along the $\omega$-axis and the $Y$-axis completes a right handed set of axes. This reference system is identical to that used in the CAD4Nonius diffractometer. Consider a hexagonal crystal which has been oriented about its $c^{*}$-axis on the goniometer head attached to the $\phi$-circle of the diffractometer by means of the goniometer arcs such that the reciprocal lattice vectors $[H K \cdot 0]^{*},\left[H^{\prime} K^{\prime} \cdot 0\right]^{*}$ and $[00 \cdot L]^{*}$, where $H=-H^{\prime}-2 K^{\prime}, K=2 H^{\prime}+K^{\prime}$, form a right handed Cartesian system and are coincident with the $X, Y$ and $Z$-axes of the laboratory frame when all the diffractometer angles are zero. The diffractometer angles $\chi, \phi$ and $\omega$ required to bring a reciprocal lattice point (relp). $H K \cdot L$ in diffracting condition for the bisecting geometry $(\psi=0)$ are given by:

$$
\begin{aligned}
& \chi_{B}=\sin ^{-1}\left(\zeta / d^{*}\right)=\cos ^{-1}\left(\xi / d^{*}\right) ; \phi=90^{\circ} \\
& \omega=\theta=\sin ^{-1}\left(\lambda d^{*} / 2\right), \text { where } \\
& \zeta=L c^{*}, \xi=\left(H^{2}+H K+K^{2}\right)^{1 / 2} a^{*}, \text { and } d^{*}=\left(\zeta^{2}+\xi^{2}\right)^{1 / 2}
\end{aligned}
$$

\section{Measurement of the intensity distribution along the streak}

In terms of the continuous variables $h_{1}, h_{2}$ and $h_{3}\left(=\zeta / c^{*}\right)$ along $a^{*}, b^{*}$ and $c^{*}$ reciprocal axes, the diffracted intensity from a faulted close-packed crystal with a repeat period of $n$-layers is given by (Wilson 1942):

$$
I(\zeta)=I\left(h_{1}, h_{2}\right) \sum_{m=-\infty}^{+\infty} J_{m} \exp \left(2 \pi i m \zeta / n c^{*}\right)
$$

Here $I\left(h_{1}, h_{2}\right)$ is a function of $h_{1}$ and $h_{2}$ which vanishes except when $h_{1}=H, h_{2}=K$, where $H$ and $K$ are the hexagonal indices with integral values. $J_{m}$ is the average of the square of the structure factor of a pair of layers separated by $m$ layer-spacings.

As is evident from (2), one should ideally measure intensities at equal intervals along a fault affected reciprocal lattice row integrated over the width of the reflection in the $a^{*}$ and $b^{*}$ directions in reciprocal space. This cannot be achieved by either of the conventional $\omega-\theta$ and $\omega-2 \theta$ scans except at $L=\zeta=0$ using $\omega-2 \theta$ scan for idealized conditions of perfectly parallel and monochromatic $x$-rays incident on a point crystal. One therefore employs the stationary crystal-stationary counter method to 
measure the point intensities at equal intervals along the $H K \cdot L$ row. In this method a slight divergence has to be introduced in the incident beam to cover the mosaic spread of the crystal. This causes the surface of the Ewald sphere to have a finite thickness and the length of the $H K \cdot L$ row intercepted by it will depend on the inclination of the row with respect to the Ewald sphere; the latter changing with $\zeta$. The counts recorded for a particular setting give a value $\int I(\zeta) \mathrm{d} \zeta$ where the integration is over the length $\Delta \zeta$ of the streak intercepted by the Ewald sphere. Thus the recorded intensities at various points along the streak correspond to variable portions of the streak. However, if we divide the measured intensities at each point by the length of the streak intercepted at that point, we shall obtain intensities corresponding to a fixed length of the streak. The necessity for such a correction factor was first realised by Pandey and Krishna (1977) while measuring the intensity distribution along streaked rows of a disordered $2 \mathrm{H}$-SiC crystal. It should be noted that due to the inherent beam divergence, this problem will also be encountered in the scan-methods, although the correction factors would be different.

\section{Derivation of the divergence-correction factor}

In this section expressions for the length of the streak $(\Delta \zeta)$, intercepted by the two extremes of the continuous range of Ewald spheres, will be derived for two cases. In case I, the streak intersects both the inner and the outer extremes of the Ewald sphere while in case II the streak is considered to be tangential to the inner extreme of the Ewald sphere. In the latter case, the effective divergence becomes less than the actual divergence.

Case I: Let $x_{i}, y_{i}, z_{i}$ be the cartesian coordinates of a relp. $H K \cdot L$ lying in a vertical plane of a hexagonal crystal in its 'standard-orientation' on the diffractometer. If the crystal is oriented about its $c$-axis on the goniometer head with the help of the goniometer arcs then $x_{i}=\xi, y_{i}=0$ and $z_{i}=\zeta$. These initial coordinates $\xi, 0, \zeta$ transform to $x_{f}, y_{f}, z_{f}$ when the relp $H K \cdot L$ is brought into a diffracting condition, through the following transformation matrix $(R)$

$$
R=\left|\begin{array}{lll}
\left(\cos \phi_{B} \cos \omega_{B}-\right. & \left(\cos \omega_{B} \sin \phi_{B}+\right. & \sin \omega_{B} \sin \chi_{B} \\
\left.\sin \phi_{B} \sin \omega_{B} \cos \chi_{B}\right) & \left.\sin \omega_{B} \cos \phi_{B} \cos \chi_{B}\right) & \\
-\left(\sin \omega_{B} \cos \phi_{B}+\right. & \left(-\sin \phi_{B} \sin \omega_{B}+\right. & \cos \omega_{B} \sin \chi_{B} \\
\left.\cos \omega_{B} \sin \phi_{B} \cos \chi_{B}\right) & \left.\cos \phi_{B} \cos \omega_{B} \cos \chi_{B}\right) & \\
\sin \phi_{B} \sin \chi_{B} & -\cos \phi_{B} \sin \chi_{B} & \cos \chi_{B}
\end{array}\right|
$$

Thus the final coordinates $x_{f}, y_{f}, z_{f}$ will be given by

$$
\begin{aligned}
& x_{f}=\xi\left(\cos \phi_{B} \cos \omega_{B}-\sin \phi_{B} \sin \omega_{B} \cos \chi_{B}\right)+\zeta \sin \omega_{B} \sin \chi_{B} \\
& y_{f}=-\xi\left(\cos \phi_{B} \sin \omega_{B}+\sin \phi_{B} \cos \omega_{B} \cos \chi_{B}\right)+\zeta \cos \omega_{B} \sin \chi_{B} \\
& z_{f}=\xi \sin \phi_{B} \sin \chi_{B}+\zeta \cos \chi_{B}
\end{aligned}
$$

Let $\mu$ and $v$ be the horizontal and vertical divergences in the incident beam, then the coordinates of the centers $C$ and $C_{1}$ (the middle and one extremity of the continuous range of Ewald spheres) can be written as $1 / \lambda, 0,0$ and $1 / \lambda, \mu / \lambda, v / \lambda$ respectively. The 
equations of the Ewald spheres with centres at $C$ and $C_{1}$ can be written as

$$
\begin{aligned}
& \left(x-\frac{1}{\lambda}\right)^{2}+y^{2}+z^{2}=\frac{1}{\lambda^{2}} \\
& \left(x-\frac{1}{\lambda}\right)^{2}+\left(y-\frac{\mu}{\lambda}\right)^{2}+\left(z-\frac{v}{\lambda}\right)^{2}=\frac{1}{\lambda^{2}}
\end{aligned}
$$

If the relp $H K \cdot L$ has been set to be in diffracting condition for the sphere centered at $C$ then from (4) and (5), we have on simplification:

$$
\lambda \zeta^{2}-2 \zeta \sin \omega_{B} \sin \chi_{B}-2 \xi\left(\cos \phi_{B} \cos \omega_{B}-\sin \phi_{B} \sin \omega_{B} \cos \chi_{B}\right)+\lambda \xi^{2}=0
$$

Along with the relp $H K \cdot L$, another relp $H K \cdot L_{1}$ will be lying on the Ewald sphere centered at $C_{1}$ for the same setting of the crystal. Hence from (4) and (6) we have on simplification:

$$
\begin{aligned}
\lambda \zeta_{1}^{2} & -2 \zeta_{1}\left(\sin \omega_{B} \sin \chi_{B}+\mu \cos \omega_{B} \sin \chi_{B}+v \cos \chi_{B}\right)-2 \xi\left[\cos \phi_{B} \cos \omega_{B}\right. \\
& -\sin \phi_{B} \sin \omega_{B} \cos \chi_{B}-\mu\left(\sin \omega_{B} \cos \phi_{B}+\sin \phi_{B} \cos \omega_{B} \cos \chi_{B}\right) \\
& \left.+v \sin \phi_{B} \sin \chi_{B}\right]+\lambda \xi^{2}+\mu^{2} / \lambda+v^{2} / \lambda=0
\end{aligned}
$$

The length of the streak, intercepted by the Ewald sphere when the relp $H K \cdot L$ is in diffracting condition will be given by $\Delta \zeta=\left(\zeta_{1}-\zeta\right)$ which can be determined using (7) and $(8)$ as under

$$
\begin{aligned}
\lambda(2 \zeta & +\Delta \zeta) \Delta \zeta-2 \Delta \zeta \sin \omega_{B} \sin \chi_{B}-2(\zeta+\Delta \zeta)\left(\mu \cos \omega_{B} \sin \chi_{B}\right. \\
& \left.+v \cos \chi_{B}\right)+2 \xi\left[\mu\left(\cos \phi_{B} \sin \omega_{B}+\sin \phi_{B} \cos \omega_{B} \cos \chi_{B}\right)\right. \\
& \left.-v \sin \phi_{B} \sin \chi_{B}\right]+\mu^{2} / \lambda+v^{2} / \lambda=0
\end{aligned}
$$

Substituting for $\phi_{B}$ and $\omega_{B}$ from (1) in (9) and ignoring second order terms, we have

$$
\Delta \zeta=\frac{\xi\left(v \sin \chi_{B}-\mu \cos \theta \cos \chi_{B}\right)-\zeta\left(\mu \cos \theta \sin \chi_{B}+v \cos \chi_{B}\right)}{\left(\lambda \zeta-\sin \theta \sin \chi_{B}\right)}
$$

Equation (10) can be further simplified by making substitutions from (1) and the simplified expression for $\Delta \zeta$ is as under

$$
\Delta \zeta=\mu \mathrm{d}^{*} \cot \theta \cdot \operatorname{cosec} \chi_{B}=\mu \zeta \cot \theta \cdot \operatorname{cosec}^{2} \chi_{B}
$$

Equations (10) and (11) are thus the desired expressions for the correction factor for crystal mounting under consideration. It is interesting to note here that the vertical divergence has almost no influence on $\Delta \zeta$. Equation (11) can also be written in the following form:

$$
\mu=\Delta \theta
$$

since, from Bragg's equation, we know that

$$
\Delta \theta=(\Delta \zeta / \zeta) \tan \theta \sin ^{2} \chi_{B}
$$

Case II: The intersection points $H K \cdot L^{+}$and $H K \cdot L^{-}$of the streak with the inner 
Ewald sphere centered at $C$ can be determined by solving (7) and are given below:

$$
\begin{aligned}
& \zeta^{+,-}=\frac{1}{\lambda}\left[\sin \omega_{B} \sin \chi_{B} \pm\left\{\sin ^{2} \omega_{B} \sin ^{2} \chi_{B}+2 \lambda \xi\left(\cos \phi_{B} \cos \omega_{B}\right.\right.\right. \\
& \left.\left.\left.-\sin \phi_{B} \sin \omega_{B} \cos \chi_{B}\right)-\lambda^{2} \xi^{2}\right\}^{1 / 2}\right]
\end{aligned}
$$

For the streak to be tangential to the inner sphere $\zeta^{+}-\zeta^{-}=0$ i.e.,

$$
\sin ^{2} \omega_{B} \sin ^{2} \chi_{B}+2 \lambda \xi\left(\cos \phi_{B} \cos \omega_{B}-\sin \phi_{B} \sin \omega_{B} \cos \chi_{B}\right)-\lambda^{2} \xi^{2}=0
$$

On making substitutions from (1), (15) simplifies to

$$
\begin{aligned}
& \sin ^{2} \theta \sin ^{2} \chi_{B}=0 \\
& \text { i.e. } \chi_{B}=0, \text { since } \theta \neq 0
\end{aligned}
$$

This implies that the streak is tangential to the inner sphere for $\chi_{B}=0$, i.e. for any $H K \cdot 0(H-K \neq 3 N ; N$ any integer) reflection. The length of the streak intercepted by the continuous range of Ewald spheres when the relp $H K \cdot 0$ is brought into diffracting condition can be evaluated by finding out the roots $\zeta_{1_{2}}^{+}$and $\zeta_{1_{t}}^{-}$of $(8)$ in conjunction with the condition given by (16). Substituting $\chi_{B}=0$ and $\phi_{B}=-90^{\circ}$ in (8) we get

$$
\lambda \zeta_{1}^{2}-2 v \zeta_{1}-2 \xi\left(\sin \omega_{B}+\mu \cos \omega_{B}\right)+\lambda \xi^{2}+\frac{\mu^{2}}{\lambda}+\frac{v^{2}}{\lambda}=0
$$

The roots $\zeta_{1_{t}}^{+}$and $\zeta_{1_{t}}^{-}$of this equation are

$$
\zeta_{1 i}^{+,-}=\frac{1}{\lambda}\left[v \pm\left(2 \mu \sin 2 \theta_{0}-\mu^{2}\right)^{1 / 2}\right]
$$

where $\theta_{0}$ is the Bragg angle for an $H K \cdot 0$ reflection.

Thus the correction factor $\Delta \zeta_{t}=\zeta_{1_{t}}^{+}-\zeta_{1_{t}}^{-}$for the relp $H K \cdot 0$ will be given by

$$
\Delta \zeta_{t}=\frac{2}{\lambda}\left(2 \mu \sin 2 \theta_{0}-\mu^{2}\right)^{1 / 2}
$$

It is evident from the foregoing that for all $H K \cdot L$ relps with $\zeta$ lying in the range $\zeta_{1}^{+}<\zeta<\zeta_{1}^{-}$, the streak will no longer intersect the Ewald sphere centered at $C$. This implies that the effective divergence is now less than the actual divergence. Hence it is not possible to use (10) or (19) as the correction factor for $H K \cdot L$ relps with $\zeta=L c^{*}$ lying in the range $\zeta_{1_{t}}^{+}<\zeta<\zeta_{1_{t}}^{-}$, or corresponding $\chi_{B}$ lying in the range $\chi_{1_{t}}^{+}<\chi_{B}<\chi_{1_{t}}^{-}$, even if one uses slits wide enough to record the entire streak intercepted by the Ewald sphere. It is interesting to note that the vertical as well as horizontal divergence i.e. $v$ and $\mu$ fix the limits $\zeta_{1}^{+}$and $\zeta_{1}$, of the bad region for data collection.

\section{Influence of azimuthal rotation on the intensity of diffuse reflections}

During the collection of intensity data for diffuse $10 \cdot \mathrm{L}$ reflections of a faulted $2 \mathrm{H}-\mathrm{SiC}$ crystal, an anomalous continuous variation in the intensity was observed by Pandey and Krishna (1977) on rotating the crystal through different azimuth angles $(\psi)$ around the scattering vector. The total number of counts changed by as much as a factor of 10 for the same $10 \cdot L$ reflection. Any change in the intensity of a reflection during 
azimuthal rotation of a normal crystal is usually attributed to change in absorption, anisotropic extinctions and multiple scattering. However, none of these can account for the large continuous variation in intensity. It was also reported that the fault-unaffected sharp reflections like $11 \cdot L$ showed only a small variation in intensity which could be attributed to differences in absorption and other usual factors. Photographic records of a $10 \cdot L$ reflection taken for different $\psi$ revealed that different amounts of streak were recorded at different azimuth settings. This implied that the length of the streak along the $10 \cdot L$ row intercepted by the Ewald sphere changes with $\psi$ on account of the incident beam divergence. The mathematical treatment given in the previous sections can be used to calculate the length of the streak intercepted by the Ewald sphere on azimuth rotation of a disordered crystal.

Let us consider a disordered hexagonal crystal mounted about its $c$-axis on the goniometer head of the diffractometer such that the vectors $a^{*}, b$ and $c^{*}$ form a right handed cartesian coordinate system. To simplify the mathematical treatment, we shall calculate the length of the streak $(\Delta \zeta)$ intercepted by the Ewald sphere for a 10.0 reflection as a function of the azimuth angle $\psi$. For the crystal orientation under consideration, 10.0 reflection is an 'equatorial reflection' since $\chi_{B}=0$. For an equatorial reflection the diffractometer angles in the general setting $(\psi \neq 0)$ are given by the following equation

$$
\chi= \pm \psi, \phi=\phi_{B} \mp 90 \text { and } \omega= \pm 90+\theta
$$

From (1), we know that $\phi_{B}=-90^{\circ}$, and hence the diffractometer angles will be given by (taking lower sign):

$$
\chi=-\psi, \phi=0, \text { and } \omega=-(90-\theta)
$$

Substituting for $\chi, \phi$ and $\omega$ from the above equation in (9) we get after simplification

$$
\begin{aligned}
& \lambda(2 \zeta+\Delta \zeta) \Delta \zeta-2 \Delta \zeta \cos \theta \sin \psi-2(\zeta+\Delta \zeta)(-\mu \sin \theta \sin \psi+v \cos \psi) \\
& -2 \xi \mu \cos \theta+\frac{\mu^{2}}{\lambda}+\frac{v^{2}}{\lambda}=0 .
\end{aligned}
$$

For the 10.0 reflection, $\zeta=0$. Putting $\zeta=0$ and ignoring the second order terms, we get the desired expression for $\Delta \zeta$ which gives the length of the streak intercepted by the Ewald sphere for the 10.0 reflection, as a function of the azimuth angle $\psi$

$$
\Delta \zeta=\mu \xi \operatorname{cosec} \psi
$$

The above equation is not valid for $\chi(=-\psi)$ values lying in the range $\chi_{1_{t}}^{+}<\chi<\chi_{1_{t}}^{-}$ where $\chi_{1_{t}}^{+}$and $\chi_{1_{t}}^{-}$correspond to $\zeta_{1_{t}}^{+}$and $\zeta_{1_{t}}^{-}$given by (18). This is because the crystal movements during azimuth rotation around the $[10.0]^{*}$ scattering vector and during the data collection under bisecting geometry for various relps along the $10 . L$ reciprocal lattice row are identical for the crystal mounting under consideration and therefore the effective divergence will be less than the actual divergence in the region $\chi_{1_{\mathrm{s}}}^{+}<\chi<\chi_{1_{\mathrm{f}}}^{-}$ thereby invalidating the applicability of (21).

\subsection{Experimental verification}

In order to verify the correctness of (21), which will in turn validate the mathematical treatment employed for deriving the divergence correction factors, a $2 \mathrm{H}$-SiC crystal 
was mounted on an Enraf Nonius 4-circle single crystal diffractometer. The crystal was needle-shaped, approximately $0.5 \mathrm{~mm}$ thick at the base and about $1 \mathrm{~mm}$ in length. The crystal was annealed for $16 \mathrm{hr}$ at $2273 \mathrm{~K}$ in vacuum and had transformed partially towards a $6 H(A B C A C B, \ldots)$ structure. This transformation is known to commence with a non-random insertion of layer displacement faults giving rise to sharp as well as diffuse $x$-ray reflections superimposed on characteristic diffuse streaks on the $x$-ray diffraction photographs (for details, see Pandey 1981). To collect the intensity data, the crystal was oriented about its $c$-axis on the goniometer head attached to the $\phi$-circle using the goniometer arcs. In the standard orientation of the crystal the $a^{*}, b$ and $c^{*}$ vectors formed a right handed coordinate system coincident with the coordinate system attached to the diffractometer. Sharp reflections with $H-K=0 \bmod 3$ were used to define the orientation matrix and the following hexagonal cell parameters were obtained after refinements:

$$
a=3.0763 \AA ; b=3.0763 \AA ; c=5.0480 \AA .
$$

Filtered $\operatorname{MoK} \alpha_{1}$ radiation was used to record the intensity diffracted by the crystal. The incident beam divergence was fixed by a collimator hole of $0.4 \mathrm{~mm}$ and collimatorcrystal distance of $47 \mathrm{~mm}$. A wide slit with horizontal and vertical openings of 9 and $2 \mathrm{~mm}$ respectively was used in front of the detector at a distance of $173 \mathrm{~mm}$ from the crystal.

The relp 10.0 can be brought into diffracting condition by adjusting the $\theta$ and $\omega$ values corresponding to $\chi_{B}=0$ and $\phi_{B}=-90^{\circ}$ in the bisecting geometry. When the crystal was rotated about the scattering vector $[10 \cdot 0]^{*}$, the $c^{*}$-direction was always contained in the plane of the $\chi$-circle with diffractometer angles given by (20). Figure 1 depicts the observed variation in intensity of the 10.0 reflection for azimuthal rotations at steps of $\Delta \psi= \pm 5^{\circ} . \psi$ values beyond $\pm 60^{\circ}$ where inaccessible on the diffractometer. Figure 2 depicts the variation in intensity of a 11.0 reflection, which is not affected by faulting for a similar azimuthal rotation. It is evident from figures 1 and 2 that the large anomalous variation in intensity is confined only to fault-affected reflections. The

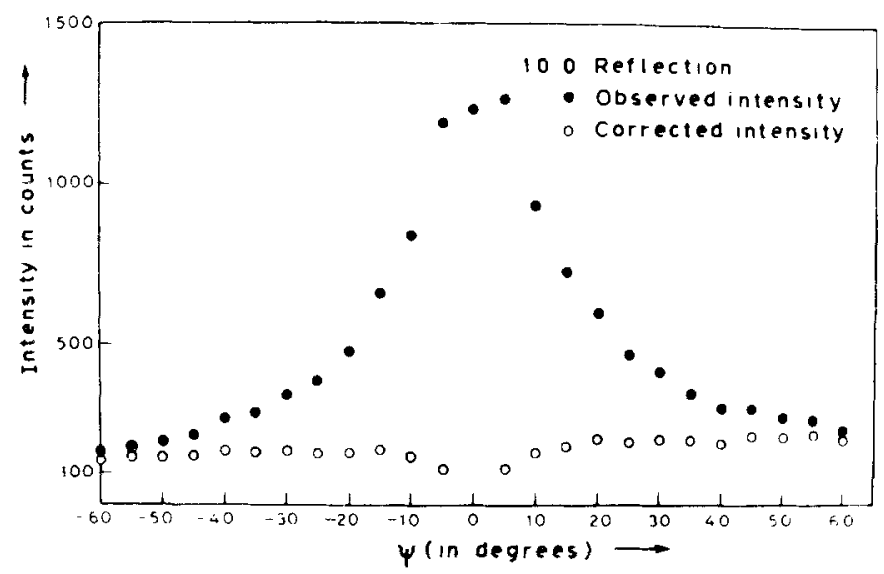

Figure 1. Variation of intensity of the 10.0 reflection of a disordered $2 \mathrm{H}-\mathrm{SiC}$ crystal as a function of the azimuth angle $\psi$ on rotating the crystal about the scattering vector. Filled circles correspond to the observed anomalous variation while open circles correspond to the variation after the application of the divergence correction factor. 


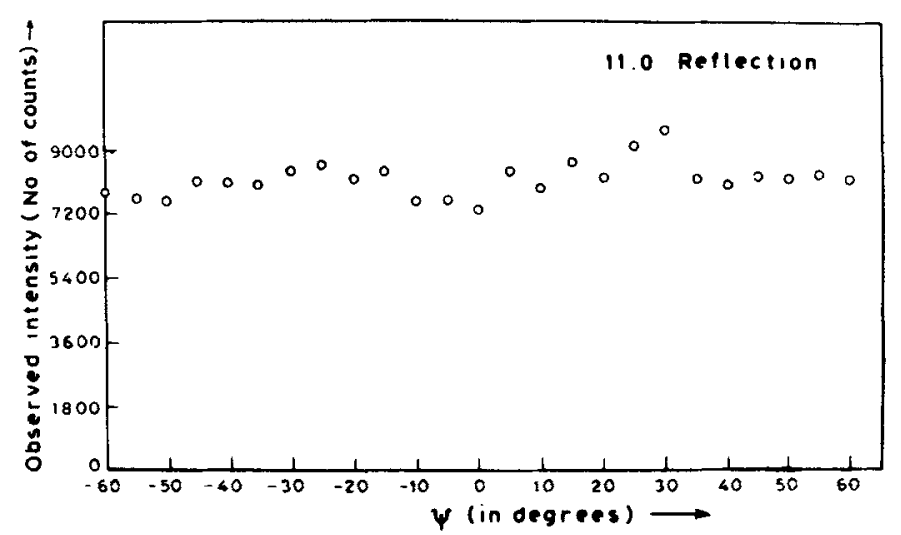

Figure 2. Variation of intensity of the 11.0 reflection of a disordered $2 H-\mathrm{SiC}$ crystal as a function of the azimuth angle $\psi$ on rotating the crystal about the scattering vector.

difference in intensities for positive and negative values of $\psi$ must be attributed to difference in absorption. The variation in intensity of the 10.0 reflection after dividing the observed intensities by $\operatorname{cosec} \psi$ is also given in figure 1 . A comparison of the observed and corrected intensities given in figure 1 shows that except for the region $-10^{\circ} \leqslant \psi \leqslant+15^{\circ}$, the correction factor given by (21) works remarkably well. The region $-10^{\circ} \leqslant \psi \leqslant+15^{\circ}$ actually corresponds to the situation for which the effective divergence is less than the actual divergence discussed earlier. The exact limits of this bad region can be calculated from (18) on substituting the following constants:

$$
\theta_{0}=7.65^{\circ} ; \mu=v=\frac{0.4}{47}=0.0085 \mathrm{rad} ; \lambda=0.71069 \AA \text {. }
$$

This gives us the limits for bad region as $-0.0817 \leqslant \zeta \leqslant 0.1056$. This can be expressed in terms of the $\chi$-angle for the crystal orientation under consideration using (1). The limits so obtained are $-12 \cdot 27^{\circ} \leqslant \chi \leqslant 15.71^{\circ}$ which is in very good agreement with the limits of the region for which the correction factor given by equation (21) does not work.

\section{Discussion}

It is evident from the foregoing that the extremely large observed variation in the intensity of the 10.0 reflection on rotating the crystal about the scattering vector, is due to variations in the length of the streak intercepted by the Ewald sphere for different values of $\psi$. On dividing the observed intensity by the length $(\Delta \zeta)$ of the streak intercepted at that azimuthal angle, one obtains very small remnant variations in the intensity of the 10.0 reflection which can be attributed to change in absorption due to the irregular shape of the crystal. However, the application of the proposed correction factor $\Delta \zeta$, in converting the observed intensities corresponding to variable values of $\Delta \zeta$ to intensities corresponding to a fixed length of the streak, is based on an implicit assumption that the intensity does not vary significantly along the length of the streak 
intercepted i.e.,

$$
\int_{\Delta \zeta} I \mathrm{~d} \zeta=I \cdot \Delta \zeta
$$

For the crystal under consideration, the 10.0 reflection is not broadened by the presence of layer displacement faults (for details, see Pandey 1981) and hence the above approximation is valid. However, for measured intensities at other points along the streak, one must take into account the dependence of $I$ on $\zeta$. This requires consideration of another correction factor, which can be treated as a parameter to get the best fit between the observed intensity distribution and those calculated for a proposed model of disorder.

\section{Acknowledgements}

We are grateful to Dr Sylvain Lecoco of University of Lyon I for his invaluable assistance in single crystal diffractometry. Financial assistance from CNRS (France) and the Mineralogy-Crystallography Laboratory, University of Lyon I, to one of the authors (DP) is gratefully acknowledged. He is grateful to the staff members of the laboratory for their warm hospitality.

\section{References}

Lele S and Pandey D 1985 Mater. Sc. Forum 3143

Pandey D 1981 Proc. Indian Natn. Sci. Acad. A47 78

Pandey D and Krishna P 1977 J. Phys. D10 2057

Pandey D, Lele S and Krishna P 1980 Proc. R. Soc. London A369 435, 451, 463

Wilson A J C 1942 Proc. R. Soc. London A180 277 